Luiz Martins Junior ${ }^{1}$

Ricardo Luiz Lorenzi ${ }^{1}$

${ }^{1}$ Coordenação de Vigilância à Saúde (COVISA), Secretaria Municipal de Saúde de São Paulo.

Contato:

Luiz Martins Júnior

Rua Santa Cruz, 81 - São Paulo/SP Núcleo de Engenharia de Segurança e Medicina do Trabalho - NESMT

- $2^{\circ}$ Andar - CEP: 04121-000

E-mail:

luizmartins@crt.saude.sp.gov.br

\section{Acidente químico com dióxido de enxofre em um popu- loso distrito de uma grande metrópole: cenários de ex- posição a partir de um modelo Gaussiano de dispersão}

Chemical accident with sulfur dioxide in a densely populated metropolitan district - exposure scenarios using a Gaussian

dispersion model

\section{Resumo}

O artigo relata a ocorrência de um acidente químico maior em um distrito populoso de uma grande metrópole, envolvendo a emissão de dióxido de enxofre de uma planta de produção de ácido sulfúrico. Discutem-se alguns cenários possíveis de exposição da população do entorno, cotejando-se valores estimados da concentração de dióxido de enxofre com valores referenciais da legislação vigente. $\mathrm{O}$ artigo conclui que a construção de tais cenários pode ajudar na análise do risco a que essas populações estão expostas e na tomada de decisões que objetive seu controle.

Palavras-chaves: saúde ambiental, poluição do ar, exposição ambiental, compostos de enxofre, poluição industrial / análise e efeitos adversos.

\section{Abstract}

The article reports a major chemical accident occurred in a densely populated metropolitan district involving sulfur dioxide emissions of sulfuric acid plants. Possible scenarios for the exposure of neighborhood population are discussed and sulfur dioxide concentration estimates are compared with current legal reference values. The article concludes that building such scenarios can be helpful to analyze the risk of population exposure and it may become a useful decision-making tool on risk control.

Keywords: environmental health, air pollution, environmental exposure, sulfur compounds, industrial pollution / analysis and adverse effects. 


\section{Introdução}

Acidentes envolvendo substâncias perigosas nas atividades de transporte, armazenamento e produção industrial de produtos químicos constituem sério risco à saúde e ao meio ambiente. O crescimento dessas atividades em nível global têm provocado aumento no número de seres humanos expostos aos seus riscos - trabalhadores e comunidades (FREITAS, PORTE \& GOMES, 1995). Ao mesmo tempo, intensificase a transferência de riscos relacionados à atividade produtiva e ao meio ambiente de países de primeiro mundo para os países em desenvolvimento (JEYARATNAM, 1994).

Consideram-se acidentes maiores ocorrências como emissão, incêndio ou explosão envolvendo uma ou mais substâncias químicas perigosas, resultando de um desenvolvimento incontrolável no curso da atividade industrial, conduzindo a sérios perigos ao homem e ao meio ambiente, imediatos ou a longo prazo, interna e externamente ao estabelecimento (EC, 1982). Outros autores consideram acidentes químicos ampliados os eventos agudos, tais como explosões, incêndios e emissões, individualmente ou combinados, envolvendo uma ou mais substâncias perigosas com potencial de causar simultaneamente múltiplos danos ao meio ambiente e à saúde dos seres humanos expostos (FREITAS, PORTE \& GOMES, 1995).

Aditivamente às emissões provenientes de fontes fixas, são relevantes, do ponto de vista da saúde pública, as emissões de veículos automotores na produção de efeitos agudos ou crônicos sobre a saúde humana.

\section{Caso motivador do estudo apresentado}

A indústria estudada situa-se em uma grande metrópole do Estado de São Paulo, no Brasil, numa área classificada como Zona Industrial pelo Plano Diretor Municipal, que tem em sua proximidade bairros residenciais com expressiva densidade populacional, além de intensa circulação de pessoas. A população no distrito atingido é estimada em mais de 90.000 habitantes e, num raio de $1,5 \mathrm{~km}$, possui uma densidade populacional estimada em cerca de 7.000 habitantes $/ \mathrm{km}^{2}$.

A cartografia da região do entorno da indústria mostra uma elevação de cerca de quatro metros do terreno a partir da fonte emissora até a $300 \mathrm{~m}$ da fonte, prosseguindo em elevação em direção ao bairro na medida em que se afasta da fonte emissora até a elevação de sete metros a $500 \mathrm{~m}$ da fonte.

As características geográficas, como a proximidade da fonte emissora a áreas densamente povoadas, o aclive do terreno em direção à região de adensamento populacional, aliadas às condições climáticas, como velocidade e direção predominante dos ventos, existência ou não de inversão térmica, contribuem para a gravidade de um acidente maior, como o do relato de caso a seguir.

Em 2006, durante a retomada da produção, ocorreu uma emissão de gás dióxido de enxofre $\left(\mathrm{SO}_{2}\right)$ que, ultrapassando as fronteiras da fábrica de ácido sulfúrico, atingiu a população do entorno. Várias pessoas apresentaram agravos à saúde relacionados ao caso, compreendendo irritação das vias aéreas, desmaios, vômitos e enjôos, demandando atendimento hospitalar de emergência em hospitais da região. Também foram acionados o Serviço de Atendimento Médico de Urgência (SAMU) e o Corpo de Bombeiros.

\section{Descrição do processo produtivo}

Trata-se de uma planta industrial de fabricação de ácido sulfúrico 98\% a partir da queima de enxofre elementar com capacidade de produção inicial de 600 toneladas por dia.

\section{Fusão e combustão do enxofre}

A fabricação de ácido sulfúrico inicia-se com a fusão de enxofre elementar. O enxofre fundido é filtrado e sofre oxidação conforme a equação:

$$
\mathrm{S}+\mathrm{O}_{2} \longrightarrow \mathrm{SO}_{2}
$$

O dióxido de enxofre, então, é obtido por meio da queima do enxofre elementar de forma a produzir um gás contendo uma mistura de $\mathrm{SO}_{2}$ (8\% a 11\%), Nitrogênio e Oxigênio.

\section{Conversão catalítica do $\mathrm{SO}_{2}$}

A conversão de $\mathrm{SO}_{2}$ em $\mathrm{SO}_{3}$ é promovida com auxílio do catalisador Pentóxido de Vanádio $\left(\mathrm{V}_{2} \mathrm{O}_{5}\right)$. A temperatura da mistura gasosa aumenta com a reação. Uma máxima conversão a $\mathrm{SO}_{3}$ requer limitação da temperatura com estágios intermediários de resfriamento. Plantas com quatro estágios de conversão têm capacidade de conversão entre $96 \%$ e 98\%. O controle de temperatura é importante para uma conversão eficiente (EPA, 1977). A velocidade de reação de catalisadores convencionais é praticamente nula em temperaturas inferiores a $400^{\circ} \mathrm{C}$, o que favoreceria uma maior emissão de $\mathrm{SO}_{2}$ para o meio ambiente.

\section{Sistema de dupla absorção}

Neste sistema, uma maior fração de enxofre é convertida a ácido sulfúrico, pois o $\mathrm{SO}_{3}$ formado nos primeiros estágios de conversão é removido numa torre de absorção primária e o restante retorna no último estágio de conversão para novamente ser removido na torre de absorção final, podendo se obter até 99,7\% de conversão do enxofre em ácido sulfúrico (EPA, 1977). O ácido produzido é, então, armazenado e distribuí- 
do. Deste modo, a fábrica é projetada para funcionar continuamente, 24 horas por dia.

\section{Objetivos}

Discutir a dispersão de gás $\mathrm{SO}_{2}$ emitido em um acidente químico maior ocorrido em uma indústria de fabricação de ácido sulfúrico de uma grande metrópole. Analisar possíveis cenários de exposição, selecionadas diferentes distâncias em relação à fonte emissora e cargas de enxofre prováveis. Estimar a magnitude das concentrações dos poluentes nestes locais, comparando-se as estimativas produzidas aos valores de referência dos parâmetros legais vigentes. Discutir o possível impacto à saúde da população residente e transiente e propor medidas corretivas que visem à segurança da população do entorno.

\section{Métodos}

\section{Análise da dispersão estimada de poluentes}

O modelo de dispersão Gaussiano de Pasquill (PEAVY, ROWE \& TCHOBANOGLOUS, 1985) foi construído para quatro cenários hipotéticos distintos, considerando a quantidade de reagentes utilizada na partida da fábrica, a porcentagem de absorção do $\mathrm{SO}_{2}$ esperada no processo e a distância entre a fonte emissora e o local da medida estimada. Sendo assim, obtivemos estimativas de concentração de $\mathrm{SO}_{2}$ no nível do solo para os seguintes cenários:

- O primeiro cenário estima a concentração máxima de $\mathrm{SO}_{2}$ na distância máxima em que a pluma toca o solo em uma situação de partida onde se utilize a maior carga $(6.000 \mathrm{~kg} / \mathrm{h})$ de enxofre elementar, assumindo que a conversão do $\mathrm{SO}_{2}$ gerado a $\mathrm{SO}_{3}$ é insignificante devido à baixa temperatura no leito do conversor. É improvável sua ocorrência devido à existência de pré-aquecimento dos leitos do conversor.
- O segundo cenário refere-se à concentração máxima de $\mathrm{SO}_{2}$ na distância máxima em que a pluma toca o solo com a utilização da maior carga de enxofre elementar, assumindo, entretanto, uma conversão de $\mathrm{SO}_{2}$ a $\mathrm{SO}_{3}$ de $50 \%$.

- O terceiro cenário desenvolve a estimativa da concentração de $\mathrm{SO}_{2}$ à distância pré-definida de 300 $\mathrm{m}$, utilizando uma menor carga de enxofre elementar e assumindo uma conversão de $\mathrm{SO}_{2}$ a $\mathrm{SO}_{3}$ de $50 \%$.

- O quarto cenário estima a concentração de $\mathrm{SO}_{2}$ à distância de $500 \mathrm{~m}$ da fonte emissora e na direção do vento utilizando uma menor carga de enxofre elementar e assumindo uma conversão de $\mathrm{SO}_{2}$ a $\mathrm{SO}_{3}$ de $50 \%$.

Utilizamos o cálculo estequiométrico para estimar a quantidade de gás $\mathrm{SO}_{2}$ produzida pela combustão do enxofre utilizando a seguinte equação:

\begin{tabular}{|c|c|c|c|}
\hline & S & $\mathrm{O}_{2}$ & $\mathrm{SO}_{2}$ \\
\hline (1) & $32 \mathrm{~g}$ & $32 \mathrm{~g}$ & $64 \mathrm{~g}$ \\
\hline (2) & $3 \times 10^{9} \mathrm{mg}$ & $3 \times 10^{9} \mathrm{mg}$ & $6 \times 10^{9} \mathrm{mg}$ \\
\hline (3) & $6 \times 10^{9} \mathrm{mg}$ & $6 \times 10^{9} \mathrm{mg}$ & $12 \times 10^{9} \mathrm{mg}$ \\
\hline
\end{tabular}

Assumimos que a carga de enxofre elementar compatível à partida desta fábrica encontra-se entre $3.000 \mathrm{~kg} / \mathrm{h}$ e $6.000 \mathrm{~kg} / \mathrm{h}$, para fins deste cálculo estequiométrico, como podemos visualizar acima (Linhas 2 e 3). Assim, a quantidade de gás $\mathrm{SO}_{2}$ que é produzida na partida e se dirige ao conversor é da ordem de $6.000 \mathrm{~kg} / \mathrm{h}$ a $12.000 \mathrm{~kg} / \mathrm{h}$.

Consideramos que, a taxa média de conversão de $\mathrm{SO}_{2}$ a $\mathrm{SO}_{3}$ esperada na partida da planta é $50 \%$.

\section{Classes de Estabilidade de Pasquill}

Para estimar a dispersão do gás $\mathrm{SO}_{2}$ na atmosfera, consideraremos as classes de estabilidade segundo Pasquill (1961) (Quadro 1).

Quadro 1 Classes de Estabilidade de Pasquill - Gifford

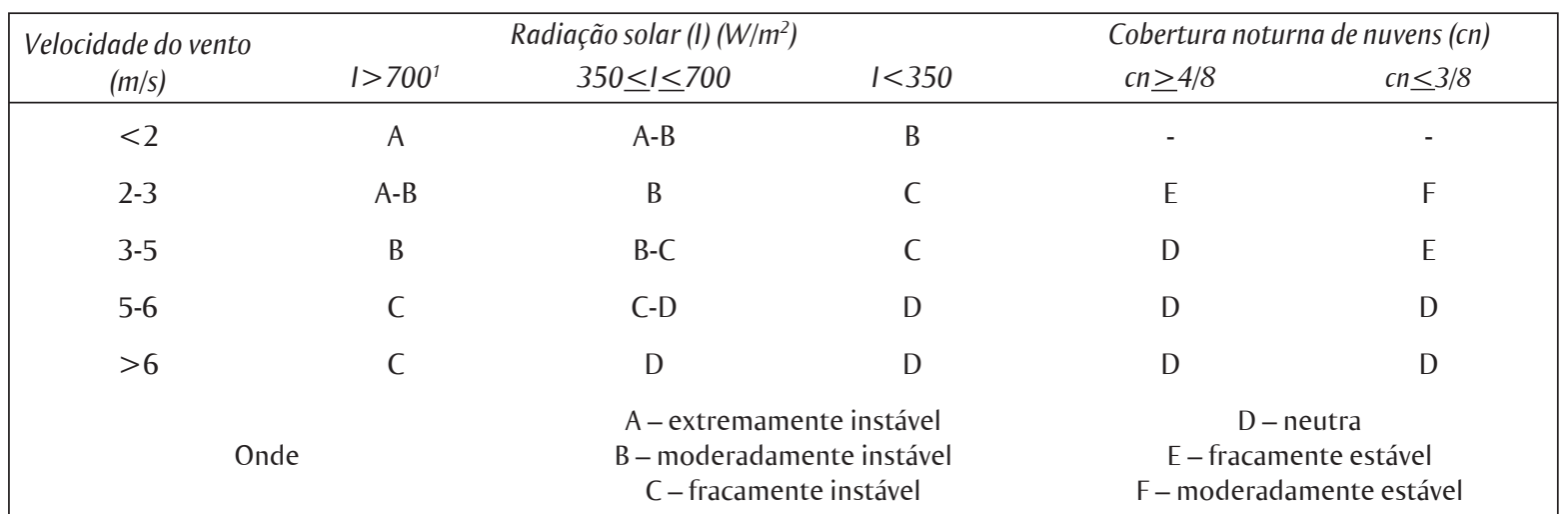

Fonte: PEAVY, H. S. et al. Environmental Engineering. McGraw-Hill, 1985.

Notas: 1 - Forte insolação (I>700) corresponde a um ângulo de elevação solar acima do horizonte de $60^{\circ}$ ou mais. Fraca insolação corresponde a um ângulo de elevação solar acima do horizonte entre $15^{\circ}$ e $35^{\circ}$. 
Equação de Pasquill

$\mathrm{C}(\mathrm{x}, 0,0, \mathrm{H})=\frac{\mathrm{Q}}{\pi \cdot \sigma_{y} \cdot \sigma_{z} \cdot u} \cdot e\left[-\frac{1}{2}\left(\frac{\mathrm{H}}{\sigma_{2}}\right)^{2}\right]$

Onde:

C - Concentração do poluente em qualquer ponto de coordenadas $(\mathrm{x}, \mathrm{y}, \mathrm{z}), \mathrm{g} / \mathrm{m}^{3}$

$\mathrm{Q}$ - Taxa de emissão uniforme, g/s

H - Altura efetiva de emissão dos poluentes, m

u - Velocidade média do vento, $\mathrm{m} / \mathrm{s}$

$\sigma_{\mathrm{z}}$ - Coeficiente de dispersão horizontal, m

$\sigma_{\mathrm{y}}$ - Coeficiente de dispersão vertical, $\mathrm{m}$

$\mathrm{x}, \mathrm{y}$ e $\mathrm{z}$ - Coordenadas do ponto

\section{Padronizações para emissões de $\mathrm{SO}_{2}$}

Padronizações internacionais admitem emissões de $\mathrm{SO}_{2}$ abaixo de $2 \mathrm{~kg}$ por tonelada métrica de ácido produzido, sendo a produção expressa como 100\% de $\mathrm{H}_{2} \mathrm{SO}_{4}$ (EPA, 1977).
A legislação nacional, por meio da Resolução CONAMA n ${ }^{\circ} 3$ (Brasil, 1990), estabelece, em relação ao controle de poluentes, dois padrões de qualidade do ar: padrões primários de qualidade do ar são as concentrações de poluentes que, quando ultrapassadas, podem acarretar danos à saúde da população; padrões secundários de qualidade do ar são as concentrações de poluentes abaixo das quais se espera o mínimo efeito sobre a saúde da população, da fauna e da flora. Define para o dióxido de enxofre $\left(\mathrm{SO}_{2}\right)$ : padrão primário, concentração média aritmética anual de 80 (oitenta) microgramas por metro cúbico de ar, concentração média de 24 (vinte e quatro) horas de 365 (trezentos e sessenta e cinco) microgramas por metro cúbico de ar, que não deve ser excedida mais de uma vez por ano; padrão secundário, uma concentração média aritmética anual de 40 (quarenta) microgramas por metro cúbico de ar e concentração média de 24 (vinte e quatro) horas de 100 (cem) microgramas por metro cúbico de ar, que não deve ser excedida mais de uma vez por ano (Quadro 2). A mesma resolução também estabelece Critérios para Episódios Agudos de Poluição do Ar (Quadro 3). O padrão primário estabelecido pela norma nacional coincide com aquele da EPA (Quadro 4).

Quadro 2 Padrões nacionais de qualidade do ar, segundo Resolução CONAMA n 3 de 28/06/90

\begin{tabular}{|c|c|c|c|}
\hline Poluente & Tempo de amostragem & $\begin{array}{c}\text { Padrão primário * } \\
\left(\mu / \mathrm{m}^{3}\right)\end{array}$ & Padrão secundário *** $\left(\mu / \mathrm{m}^{3}\right)$ \\
\hline Dióxido de enxofre $\left(\mathrm{SO}_{2}\right)$ & 24 horas & $\begin{array}{c}365 \\
80\end{array}$ & $\begin{array}{c}100 \\
40\end{array}$ \\
\hline
\end{tabular}

Fonte: Modificada de CONAMA n ${ }^{\circ} 3$ (Brasil, 1990).

(1) MAA - média aritmética anual

* padrões primários de qualidade do ar são as concentrações de poluentes que, quando ultrapassadas, podem acarretar danos à saúde da população.

**** padrões secundários de qualidade do ar são as concentrações de poluentes abaixo das quais se espera o mínimo efeito sobre a saúde da população, da fauna e da flora.

Quadro 3 Critérios para episódios agudos de poluição do ar

\begin{tabular}{|lccc|}
\hline & & Níveis & Emergência \\
\hline Dióxido de enxofre $\left(\mathrm{SO}_{2}\right)\left(\mu / \mathrm{m}^{3}\right) 24 \mathrm{~h}$ & Atenção & Alerta & 2.100 \\
$\mathrm{SO}_{2} \times \mathrm{PTS}\left(\mu / \mathrm{m}^{3}\right) 24 \mathrm{~h}$ & 800 & 1.600 & 393.000 \\
\hline
\end{tabular}

Fonte: Modificado de CONAMA no 3 (Brasil, 1990).

Quadro 4 Padrões de qualidade do ar para os principais poluentes segundo a Environmental Protection Agency (EPA) dos Estados Unidos da América

\begin{tabular}{|ccc|}
\hline Poluente & Padrão primário $\left(\mu / \mathrm{m}^{3}\right)$ & Tempo de amostragem \\
\hline & $80(0,03 \mathrm{ppm})$ & Média aritmética anual \\
Dióxido de enxofre $\left(\mathrm{SO}_{2}\right)$ & $365(0,14 \mathrm{ppm})$ & Nível máximo em $24 \mathrm{~h}$ \\
\hline
\end{tabular}

Fonte: Modificada de Bascon et al. (1996) apud Braga et al. (2002) 


\section{Resultados}

Cenário 1: Estimativa da concentração máxima no nível do solo a distância máxima em que a pluma toca o solo com carga inicial de enxofre igual a $6.000 \mathrm{~kg} / \mathrm{h}$ e nenhuma conversão de $\mathrm{SO}_{2}$ a $\mathrm{SO}_{3}$.

Considerando a velocidade dos ventos de $5,5 \mathrm{~m} / \mathrm{s}$, admitida a altura da chaminé de $40 \mathrm{~m}$. Supondo a utilização de carga de $6.000 \mathrm{~kg} / \mathrm{h}$ de enxofre e formação de $12.000 \mathrm{~kg} / \mathrm{h}$ de $\mathrm{SO}_{2}$ para o conversor com temperaturas inferiores a $400^{\circ} \mathrm{C}$, considerando que uma quantidade insignificante de $\mathrm{SO}_{2}$ tenha sido convertida.

Para o problema em questão, consideraremos a utilização da classe "C", fracamente instável. Assim, para uma altura da chaminé $\mathrm{H}=40 \mathrm{~m}$, temos $\sigma_{\mathrm{z}} / \sigma_{\mathrm{y}}=$ constante $\longrightarrow \sigma_{\mathrm{z}}=0,707 \times \mathrm{H}=0,707 \times 40 \longrightarrow$ $\sigma_{\mathrm{z}}=28,8 \mathrm{~m}$.

Para $\sigma_{\mathrm{z}}=28,8 \mathrm{~m}$, consultando o Ábaco para determinação do coeficiente de dispersão vertical $\left(\sigma_{\mathrm{Z}}\right)$ segundo Pasquill (PEAVY, ROWE \& TCHOBANOGLOUS, 1985), encontramos $X_{\text {máx }}=400 \mathrm{~m}$, ou seja, a máxima distância em que a pluma toca o solo e que corresponde à máxima concentração ao nível do solo.

Para $\mathrm{X}_{\text {máx }}=400 \mathrm{~m}$, consultando o Ábaco para determinaçã̃o do coeficiente de dispersão horizontal $\left(\sigma_{\mathrm{y}}\right)$ segundo Pasquill (PEAVY, ROWE \& TCHOBANOGLOUS, 1985), encontramos $\sigma_{\mathrm{y}}=50 \mathrm{~m}$.

Taxa de emissão de $\mathrm{SO}_{2} \longrightarrow \mathrm{Q}=12 \times 10^{9} \mathrm{mg} / \mathrm{h}$ $=\longrightarrow \mathrm{Q}=12 \times 10^{3} \mathrm{~kg}$ de $\mathrm{SO}_{2} / \mathrm{h} \times \mathrm{h} / 3600 \mathrm{~s} \times 1000$ $\mathrm{g} / \mathrm{kg} \longrightarrow \mathrm{Q}=3333,33 \mathrm{~g} / \mathrm{s}$.

$$
\begin{aligned}
\mathrm{C}_{\text {máx }}= & (3333,33 / \pi \times 5,5 \times 28,8 \times 50) \times \operatorname{Exp}-1 / 2 \\
& {[40 / 28,8]^{2} } \\
\mathrm{C}_{\text {máx }}= & 50.175 \mu \mathrm{g} / \mathrm{m}^{3}
\end{aligned}
$$

De acordo com NIOSH Pocket Guide (CDC, 2005), $2,62 \mathrm{mg} / \mathrm{m}^{3}\left(2.620 \mu \mathrm{g} / \mathrm{m}^{3}\right)$ de $\mathrm{SO}_{2}$ equivalem a $1 \mathrm{ppm}$. Assim temos:

$$
\mathrm{C}_{\text {máx }}=19 \mathrm{ppm}
$$

Cenário 2: Estimativa da concentração máxima no nível do solo a distância máxima em que a pluma toca o solo com carga inicial de enxofre igual a $6.000 \mathrm{~kg} / \mathrm{h}$ e conversão de $\mathrm{SO}_{2}$ a $\mathrm{SO}_{3}$ em $50 \%$.

Considerando a velocidade dos ventos de $5,5 \mathrm{~m} / \mathrm{s}$, admitida a altura da chaminé de $40 \mathrm{~m}$. Supondo a utilização de carga de $6.000 \mathrm{~kg} / \mathrm{h}$ de enxofre e formação de $12.000 \mathrm{~kg} / \mathrm{h}$ de $\mathrm{SO}_{2}$ para o conversor, a uma taxa de conversão de $50 \%$, atingiríamos a taxa de $6.000 \mathrm{~kg} / \mathrm{h}$ de $\mathrm{SO}_{2}$ lançado na chaminé.

Taxa de emissão de $\mathrm{SO}_{2} \longrightarrow \mathrm{Q}=6 \times 10^{9} \mathrm{mg} / \mathrm{h}=$ $\longrightarrow \mathrm{Q}=6 \times 10^{3} \mathrm{~kg}$ de $\mathrm{SO}_{2} / \mathrm{h} \times \mathrm{h} / 3600 \mathrm{~s} \times 1000 \mathrm{~g} / \mathrm{kg}$ $\longrightarrow \mathrm{Q}=1666,66 \mathrm{~g} / \mathrm{s}$.

$$
\begin{aligned}
\mathrm{C}_{\text {máx }}= & (1666,66 / \pi \times 5,5 \times 28,8 \times 50) \times \operatorname{Exp}-1 / 2 \\
& {[40 / 28,8]^{2} }
\end{aligned}
$$

$$
\begin{aligned}
& \mathrm{C}_{\text {máx }}=25.087 \mu \mathrm{g} / \mathrm{m}^{3} \\
& \mathrm{C}_{\text {máx }}=9,5 \mathrm{ppm}
\end{aligned}
$$

Cenário 3: Estimativa da concentração de $\mathrm{SO}_{2}$ à distância pré-definida de $300 \mathrm{~m}$ da fonte.

Considerando a distância da fonte emissora de $300 \mathrm{~m}$, a velocidade dos ventos de $5,5 \mathrm{~m} / \mathrm{s}$, admitida a altura da chaminé de $40 \mathrm{~m}$, elevação do terreno de $4 \mathrm{~m}$ acima do nível da fonte emissora e uma partida com utilização de carga de $3.000 \mathrm{~kg} / \mathrm{h}$ de enxofre e formação de $6.000 \mathrm{~kg} / \mathrm{h}$ de $\mathrm{SO}_{2}$ para o Conversor. A uma taxa de conversão de 50\%, atingiríamos a taxa de $3.000 \mathrm{~kg} / \mathrm{h}$ de $\mathrm{SO}_{2}$ lançado na chaminé.

Taxa de emissão de $\mathrm{SO}_{2} \longrightarrow \mathrm{Q}=3 \times 10^{9} \mathrm{mg} / \mathrm{h}=$ $\longrightarrow \mathrm{Q}=3 \times 10^{3} \mathrm{~kg}$ de $\mathrm{SO}_{2} / \mathrm{h} \times \mathrm{h} / 3600 \mathrm{~s} \times 1000 \mathrm{~g} / \mathrm{kg}$ $\longrightarrow \mathrm{Q}=833,33 \mathrm{~g} / \mathrm{s}$.

$$
\begin{aligned}
\mathrm{C}_{\text {máx }}= & (833,33 / \pi \times 5,5 \times 25 \times 37) \times \operatorname{Exp}-1 / 2 \\
\mathrm{C}_{\text {máx }}= & 18.487 \mu \mathrm{g} / \mathrm{m}^{3} \\
\mathrm{C}_{\text {máx }}= & 7 \mathrm{ppm}
\end{aligned}
$$

Cenário 4: Estimativa da concentração de $\mathrm{SO}_{2}$ à distância pré-definida de $500 \mathrm{~m}$ da fonte emissora com carga de enxofre igual a $3.000 \mathrm{~kg} / \mathrm{h}$ e conversão de $\mathrm{SO}_{2}$ a $\mathrm{SO}_{3}$ em $50 \%$.

Considerando a distância da fonte emissora de 500 $\mathrm{m}$, a velocidade dos ventos de $5,5 \mathrm{~m} / \mathrm{s}$, altura admitida da chaminé de $40 \mathrm{~m}$, elevação do terreno de $7 \mathrm{~m}$ acima do nível da fonte emissora e uma partida com utilização de carga de $3.000 \mathrm{~kg} / \mathrm{h}$ de enxofre e formação de $6.000 \mathrm{~kg} / \mathrm{h}$ de $\mathrm{SO}_{2}$ para o Conversor. A uma taxa de conversão de 50\%, atingiríamos a taxa de $3.000 \mathrm{~kg} / \mathrm{h}$ de $\mathrm{SO}_{2}$ lançado na chaminé.

Taxa de emissão de $\mathrm{SO}_{2} \longrightarrow=3 \times 10^{9} \mathrm{mg} / \mathrm{h}=$

$\rightarrow \mathrm{Q}=3 \times 10^{3} \mathrm{~kg}$ de $\mathrm{SO}_{2} / \mathrm{h} \times \mathrm{h} / 3600 \mathrm{~s} \times 1000 \mathrm{~g} / \mathrm{kg}$ $\longrightarrow \mathrm{Q}=833,33 \mathrm{~g} / \mathrm{s}$.

$$
\begin{aligned}
\mathrm{C}_{\text {máx }}= & (833,33 / \pi \times 5,5 \times 37 \times 60) \times \operatorname{Exp}-1 / 2 \\
& {[33 / 37]^{2} } \\
\mathrm{C}_{\text {máx }}= & 14.595 \mu \mathrm{g} / \mathrm{m}^{3} \\
\mathrm{C}_{\text {máx }}= & 5,5 \mathrm{ppm}
\end{aligned}
$$

\section{Discussão}

Um aumento de cerca de 4.000 mortes em relação à média de óbitos ocorreu após exposição a elevadas concentrações de dióxido de enxofre $\left(\mathrm{SO}_{2}\right)$ e particulados em suspensão durante episódio de inversão térmica que impediu a dispersão dos poluentes em Londres em 1952 (AMDUR et al., 1991; MAZUMDAR et al., 1982; WHO, 1979, apud ATSDR, 1998). As evidências disponíveis sugerem que o excesso de mortalidade pode ocorrer a concentrações de dióxido de enxofre maior ou igual a $500 \mu \mathrm{g} / \mathrm{m}^{3}$ (0,2 ppm) (média 
de concentração - 24 horas) em combinação com elevado nível de concentração de material particulado. A elevação da mortalidade foi atribuída à bronquite e a outros problemas respiratórios, tendo ocorrido principalmente entre idosos e indivíduos com condições cardíacas ou respiratórias pré-existentes (WHO, 1979 apud ATSDR, 1998).

Elevada mortalidade por doença cardiovascular também tem sido relatada. No Município de São Paulo, Sharovsky (2001) encontrou associação significativa entre infarto agudo do miocárdio e concentração atmosférica de $\mathrm{SO}_{2}$, aumentando-se o risco deste agravo em $3,4 \%$ para cada elevação de $10 \mu \mathrm{g} / \mathrm{m}^{3}$. De acordo com Gouveia et al. (2006), ocorre associação significativa entre o incremento de $10 \mu \mathrm{g} / \mathrm{m}^{3}$ de $\mathrm{SO}_{2}$ e o risco de internação de idosos e crianças em São Paulo por doenças respiratórias e cardiovasculares.

Considerando tais efeitos potenciais do $\mathrm{SO}_{2}$ à saúde, o modelo aqui desenvolvido estimou, para cada cenário considerado, a concentração de $\mathrm{SO}_{2}$ no nível do solo durante o processo de retomada da produção quando é prevista uma maior emissão de gases $\mathrm{SO}_{2}$. Deste modo, comparativamente aos limites previstos pela Resolução CONAMA nº 3 (Quadros 2 e 3), obtivemos:

Para o Cenário 1: Concentração maior que 137 vezes o padrão primário, $171 \%$ da média aritmética anual e maior que 24 vezes o nível de emergência.

Para o Cenário 2: Concentração maior que 68 vezes o padrão primário, 86\% da média aritmética anual e maior que 12 vezes o nível de emergência.

\section{Referências}

ATSDR. Agency for Toxic Substances and Disease Registry. Report. Toxicological Profile for Sulfur Dioxide. p. 13. Atlanta, 1998. Available from: http:/ www.atsdr.cdc.gov/toxprofiles/tp116.pdf. Access: 12 jan. 2007.

BRAGA, A.; PEREIRA L. A. A.; SALDIVA, P. H. N. Poluição atmosférica e seus efeitos na saúde humana. In: SUSTENTABILIDADE NA GERAÇÃO E USO DE ENERGIA, UNICAMP, 18-20 de fevereiro de 2002, Campinas. Disponível em: http://www.cgu.unicamp. br/energia2020/papers/paper_Saldiva.pdf. Acesso em: 25 jan. 2007.

BRASIL. Ministério do Meio Ambiente. Conselho Nacional do Meio Ambiente. Resolução CONAMA n ${ }^{\circ}$. 3, de 28 de junho de 1990. Diário Oficial da União, Brasília, DF, 22 agosto 1990, Seção I, p. 15937-15939. Disponível em: http:/www.mma.gov.br/port/conama/ res/res90/res0390.html. Acesso em: 13 fev. 2007.
Para o Cenário 3: Concentração maior que 50 vezes o padrão primário, 63\% da média aritmética anual e maior que 9 vezes o nível de emergência.

Para o Cenário 4: Concentração maior que 40 vezes o padrão primário, 50\% da média aritmética anual e 7 vezes o nível de emergência.

Os valores estimados foram, portanto, muito elevados, podendo ser esperados efeitos à saúde tanto transitórios como permanentes (EPA, 1977).

\section{Conclusão}

A construção de cenários pode ajudar a compreender melhor o risco a que populações circunjacentes estejam expostas. O modelo utilizado permite estimar a concentração de poluentes no nível do solo de maneira que o possível impacto à saúde e ao meio ambiente possa ser avaliado, subsidiando tomada de decisões que objetive o seu controle.

Faz-se necessária a implementação de efetivas medidas de controle sobre a emissão de $\mathrm{SO}_{2}$ especialmente na retomada do processo de produção, além da monitoração contínua das emissões e uma mais rigorosa aplicação da legislação existente.

Episódios semelhantes ao relatado podem tornar a ocorrer, especialmente na partida da fábrica, o que pode representar risco à população do entorno, impondo-se a questão da proximidade destas fábricas em relação a regiões populosas.

CDC. Centers for Disease Control. National Institute for Occupational Safety and Health. NIOSH Pocket Guide to Chemical Hazards - Sulfur Dioxide. Cincinnati, 2005. Available from: http://www.cdc. gov/niosh/npg/. Access: 15 fev. 2007.

EC. European Community. Directive 82/501/EEC (“Seveso I”). Available from: http://www.ess.co.at/ HITERM/REGULATIONS/82-501-eec.html. Access: 10 mar. 2007.

EPA. U.S. Environmental Protection Agency. Emission Standards and Engineering Division. Final Guideline Document: Control of sulfuric acid mist emissions from existing sulfuric acid production units. Report. Research Triangle Park, 1977.

Available from: http://www.epa.gov/ttn/naaqs/ozone/ ctg_act/197709_sox_epa450_2-77-019_sulfuric_ acid_mist.pdf. Access: 13 dez. 2006.

FREITAS, C. M.; PORTE, M. F. S.; GOMES, C. M. Acidentes químicos ampliados: um desafio para a 
saúde pública. Rev. Saúde Públ., São Paulo, v. 29, n. 6, p. 503-514, 1995.

GOUVEIA, N. et al. Hospitalizações por causas respiratórias e cardiovasculares associadas à contaminação atmosférica no Município de São Paulo, Brasil. Cad. de Saúde Pública, Rio de Janeiro, v. 22, n. 12, p. 2669-2677, 2006.

JEYARATNAM, J. Transfer of Hazardous Industries. In: PEARCE, N. et al. Occupational Cancer in Developing Countries. Lyon: IARC Scientific Publ., 1994. p. 23-29.
PASQUILL, F. The estimation of the dispersion of windborne material. The Meteorological Magazine, v. 90, n. 1063, p. 33-49, 1961.

PEAVY, H. S.; ROWE, D.; TCHOBANOGLOUS, G. Meteorology and natural purification process. In: Environmental Engineering. New York: McGraw-Hill, 1985. p. 502-503.

SHAROVSKY, R. Efeitos da temperatura e poluição do ar na mortalidade por infarto agudo do miocárdio no município de São Paulo. 2001. 86 f. Tese (Doutorado em Cardiologia) - Faculdade de Medicina da Universidade de São Paulo, São Paulo, 2001. 